\title{
Computer-Aided Simulation Using Finite Element Analysis of Protect Against to Coronavirus (COVID-19) of Custom-Made New Mask Design
}

\author{
Kadir Gök ${ }^{1}$ (1) $\cdot$ Akil Birkan Selçuk ${ }^{1} \cdot$ Arif Gök $^{2}$
}

Received: 8 December 2020/ Accepted: 23 February 2021/Published online: 23 March 2021

(C) The Indian Institute of Metals - IIM 2021

\begin{abstract}
The Covid-19 virus has been recognized by the World Health Organization to be creating Pandemic worldwide. It has been determined that the virus spreads very quickly and has a fatal risk in people with chronic disease. As the virus vaccine and medications to be used for treatment are not fully developed, alternative ways to protect human being from the virus are being investigated. In this study, a custom-made mask design was developed to prevent infection of the virus and computer-aided simulations were performed. Three-dimensional model of the custom-made face mask was created with the SolidWorks software. The analyses were performed using Ansys Workbench Explicit Dynamics module. The load, boundary conditions and material were defined in the Ansys Workbench. As a result of the simulations, it has been determined that custom-made designed masks have a much lower risk of virus infection than conventional masks.
\end{abstract}

Keywords COVID-19 - Reverse engineering . Custom-made mask design · Additive manufacturing · Computer-aided simulation

Kadir Gök

kadir.gok@bakircay.edu.tr

1 Department of Biomedical Engineering, Engineering and Architecture Faculty, Izmir Bakircay University, Izmir, Turkey

2 Department of Industrial Design, Architecture Faculty, Kutahya Dumlupinar University, Kutahya, Turkey

\section{Introduction}

Coronavirus disease (COVID-19) is an infectious disease caused by a new virus. The disease leads to a respiratory problem (such as the flu), along with symptoms such as dry cough, fever and difficulty in breathing in more serious cases. The Coronavirus-induced Covid-19 disease was declared as a pandemic by the World Health Organization (WHO) as of March 11. With its simplest definition, pandemic can be defined as the name given to infectious diseases that threaten many people simultaneously in the world. Within the scope of the struggle against pandemic, quarantine measures have been started in whole or in part, on a city or country scale. COVD-19 cases show that its spread is not limited to the respiratory tract [1]. Eye exposure may be considered as a way of getting infected with viruses as well [2]. Other study has stated the susceptibility of SARS-CoV-2 to transmit directly and/or indirectly through saliva during medical procedures [3]. Moreover, SARS-CoV-2 has been detected in fecal swabs of patients, indicating the possibility of fecal-oral route transmission [4-6]. The risk related to dental care settings is invariably not less than the mentioned routes referring to its procedures specificity involving face-to-face communication with patients and frequent contact with saliva, blood and other fluids [7-9].

Our primary purpose is to prevent quick spreading of this infectious outbreak. To achieve this goal, it is necessary to isolate infected people from healthy people. It is very difficult to take personal or social quarantine precautions applied in the spread of this outbreak. Firstly, the protection of high-risk health personnel within the scope of combating the epidemic is of great importance to maintain a healthy treatment process in the society. One of the most important ways to protect from virus is to use protective 
equipment. The use of masks is very important among the protective equipment. The protection against viruses by using classic disposable masks with a specified lifetime can sometimes be insufficient.

Recently, solving problems in engineering or health processes based on the finite element analysis (FEA) or Computational Fluid Dynamics (CFD) in a computer environment has become very important in terms of both reducing the higher costs of the experimental method and efficiency. Solving problems are analytically quite complex in engineering or health processes. Especially, analytical, or experimental methods may remain inadequate to determine these processes. In similar cases, CFD and FEA occur as an alternative solution. However, to obtain a good result from the method, the correct load and boundary conditions must be defined firstly. Otherwise, they may lead to undesirable results [10]. FEA is used to solve processes such as metal turning, bone drilling and bone screwing process, fatigue behaviour of implant materials, optimal configuration of implant materials [10-16].

There are some studies on this subject in the literature. Sun et al. [17] have proposed a method to solve the problem of 3D silicone mask presentation attacks by employing polarization MWIR imaging. The method uses a polarization MWIR imaging system to capture a set of data without the need for visible or NIR light sources. Li et al. [18] have addressed the problem of 3D face mask PAD from the viewpoint of intrinsic image analysis and they have designed a new feature for reflectance characteristic description. Jia $S$ et al. [19] have summarized the advances of 3D mask spoofing and anti-spoofing works over the past decade. Swennen et al. [20] have presented a proof of concept and prototype of a reusable custom-made threedimensionally printed face mask, based on individual facial scanning, three-dimensional modelling, and three-dimensional printing, that can be adopted and used worldwide in case of need. The numerous inquiries and questions such as technical factors, testing recommendations and characterization methods and biological concerns such as biocompatibility and sterilization for the AM manufactured medical devices are addressed by Tarfaoui et al. [21].

Agrawal and Bhardwaj estimated this volume of the air, which may help us to design ventilation of closed spaces and, consequently, reduce the spread of the disease [22]. Gandhi et al. [23] have presented a recently developed technology and device that uses olfactory sense detection using a novel device that is capable of generating precise level of smell digitally, and repeatedly in contactless manner. It has also been proposed a simple do-it-yourself (DIY) method for preparing a three-layered face mask that deploys two hydrophobic polypropylene nonwoven layers interspaced with a hydrophilic cellulosic cloth by Sarkar et al. [24].
In this study, custom-made mask design has been developed to prevent virus infection. Computer-aided simulations have been performed for both a classic mask and a custom-made mask design. To date, no study comparing the performance of classical mask and custom-made mask designs in terms of Covid-19 infection has been found in the literature. Which type of mask is more effective in preventing virus infection will be investigated by simulations.

\section{Computer-Aided Finite Element Simulation}

FEA is very important to develop optimum designs of devices. It is used as a reliable technique to verify of experimental and analytical results. In this study, the new 3D model of the custom-made mask was created with the aid of SolidWorks software. The simulations were performed by using the Ansys Workbench Explicit Dynamics module. The load, boundary conditions and material were defined in the Ansys Workbench.

\subsection{Modelling of Custom Made Mask Using RE Process}

Reverse Engineering (RE) process is used to copy the complex shapes and designs by special software and hardware. The outer geometry of a woman face was remodelled by scanning real a woman figure and RE technique was used to obtain its 3D computer-aided design (CAD) model. A 3D optical scanner having few cameras was used to scan the human face model. The custom-made mask was designed as in three-dimensional (3D) solid model using SolidWorks CAD software as shown in Fig. 1. 3D model of a classical mask was also made using Solidworks programme.

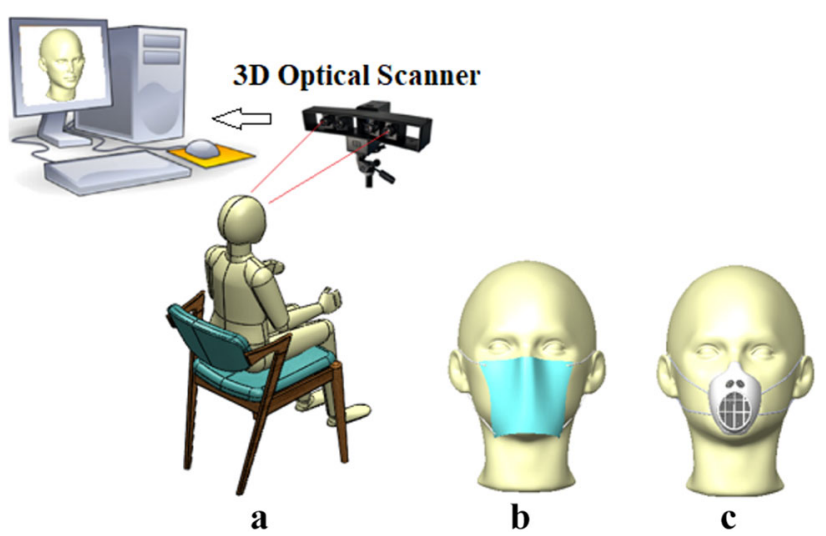

Fig. 1. 3D model of masks, a classical mask, b custom-made mask 


\subsection{Custom-Made Face Mask Using Additive Manufacturing}

Additive manufacturing, also known as 3D printing, has brought great excitement and innovation in the medical field. 3D printer was used in this research (Fig. 2a). As seen in Fig. 2b, an actual face mask was manufactured using a 3D printer (Fig. 2b).

\subsection{Loading and Boundary Conditions}

As can be seen in Fig. 3, mesh process has been performed for the classic mask and custom-made mask design and using the triangular element type in FEA. The SEA model has 138,332 nodes and 473,134 elements. The element size is selected as $5 \mathrm{~mm}$ for the human model, while the size of the other elements is automatically selected by the program. The 3D human model is fixed from the neck for FEA. It has also been fixed from ear parts of the mask. Literature research says that, water droplets formed because of coughing of a person can reach a speed of $160-800 \mathrm{~km} / \mathrm{h}$. For this process, a speed of $250 \mathrm{~km} / \mathrm{h}$ has been applied to the water droplet. Human skin has been used for the human model and Polycaprolactone (PCL) material has been used for the plastic parts of the custommade mask as it can be placed in direct contact with the skin. PCL is a biomaterial widely used in the medical field, its melting point is $100{ }^{\circ} \mathrm{C}$, it does not warp, and there is no need for a heated chamber to print it. The printed mask can also be hand-modelled to ensure a perfect fit using hot water (around $60^{\circ}$ ) [25]. The resin epoxy material has been used for replaceable filters and classical mask in FEA.

The properties of the materials used in FEA are given in Table 1. While the frictional contact type is defined between the classic mask and the human model, a frictionless contact type is also defined between both the mask and the human model by the water droplets. The type of frictional contact between the custom-made mask and the
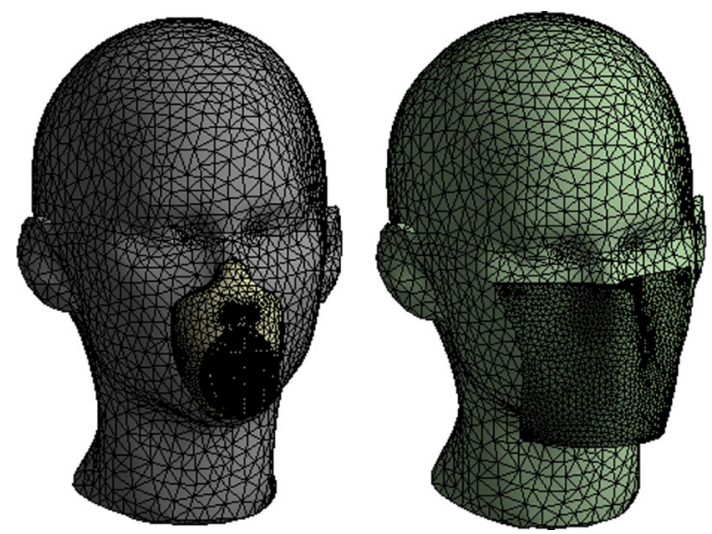

Fig. 3 Mesh of 3D model

human model, bonded contact between the filters and the mask, the frictionless contact between all the elements of the water droplets are all known. The Steinberg-Guinan strength and Shock EOS Linear models required for Explicit Dynamics analysis are obtained from the default values of each material in the Ansys Workbench material library. For dynamic simulations, the simulation time has been chosen as $0.01 \mathrm{~s}$.

\section{Results and Discussion}

The simulations were solved after the loading, and boundary conditions were defined. The results of simulations are given in Fig. 4. While water droplets that come out of the mouth, as a result of coughing of a person come out on the edges of the mask by applying high pressure in the classic mask model, these water droplets remain inside the custom-made mask and do not spread outside in any form. This can be explained by the fact that the edges of custom-made masks fit exactly to the human face. Contrary to this situation, in classical masks, the mask does not fit on
Fig. 2 a 3D printer, $\mathbf{b}$ the custom-made mask manufactured using 3D printer (The photograph in (b) belongs to the 3rd author, and permission has been obtained from him to use the photograph)
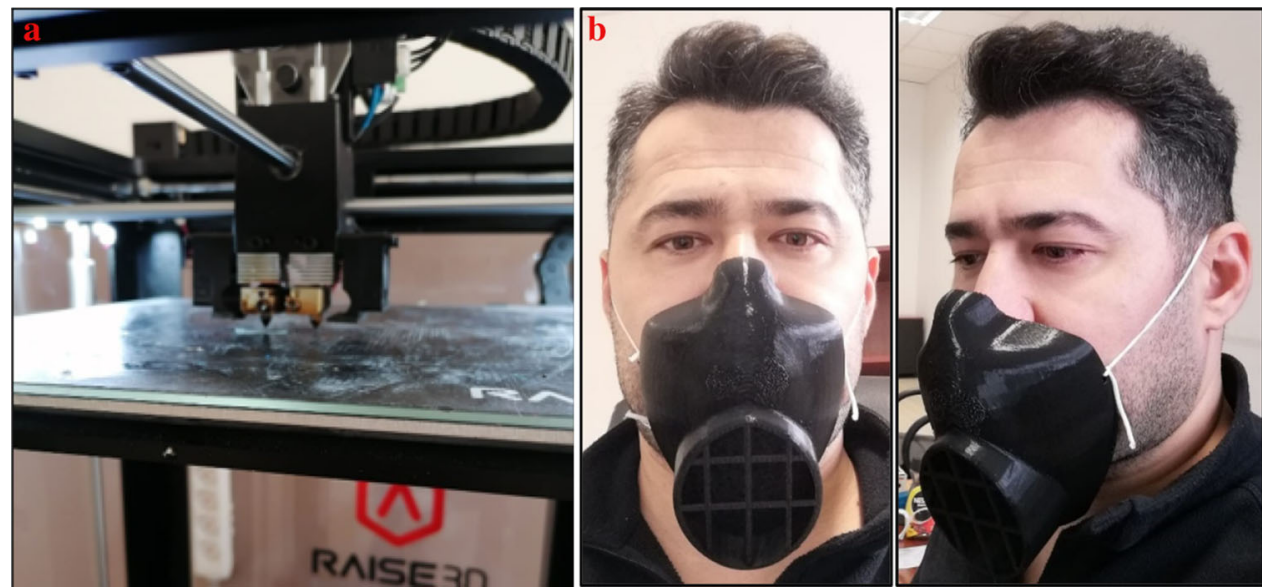
Table 1 Mechanical properties of materials used in SEA [26-28]

\begin{tabular}{lllll}
\hline Parameters & Human skin & Polycaprolactone (PCL) & Resin epoxy & Droplet \\
\hline Density $\left(\mathrm{kg} / \mathrm{m}^{3}\right)$ & 1000 & 316 & 1160 & 998 \\
Kayma Modülü (Pa) & & 22.36 & 1400 & $1 \mathrm{e}-06$ \\
Young's modulus (MPa) & 14.30 & 58.14 & 3780 & $3 \mathrm{e}-06$ \\
Poisson's ratio & 0.49 & 0.3 & 0.35 & 0.5 \\
\hline
\end{tabular}

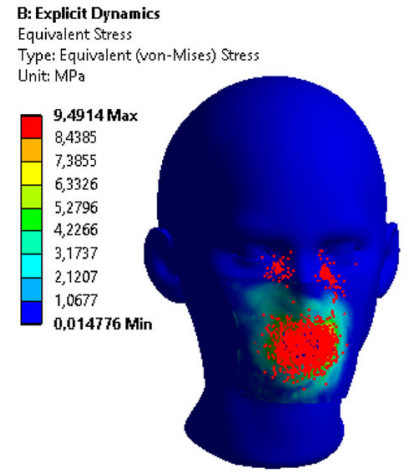

$\mathbf{a}$

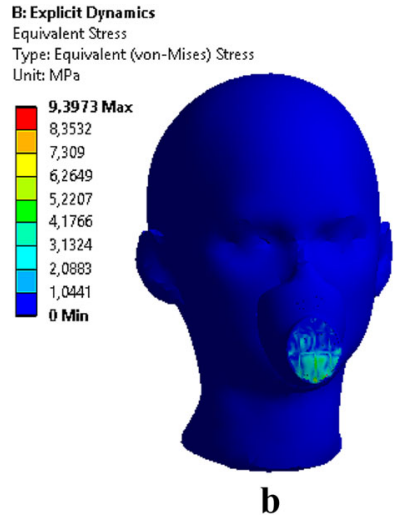

$\mathbf{b}$
Fig. 4 Stress values, a classic mask, b custom-made mask

the face of the person, and under pressure, the gaps on the edges of the mask causes water droplets to splash outside.

One of the most important ways of virus protection is protective equipment. The production of these personal protective equipment in disposable, standard and hygienic environments is of great importance in terms of virus protection. The disposable masks are very important and comfortable ones among personal equipment. It has been observed for the healthcare personnels who play an active role in the treatment of the infected patients, especially in the recent pandemic process that irritations occur on their face after a certain time. The disposable classic masks can cause droplets to come out during strong cough. This situation possesses very serious risks especially for healthcare professionals and increases the likelihood of infection among them. For this reason, custom-made masks have a very important place to protect people like healthcare personnel during normal life activities. Apart from these, classic masks cause sweating and trouble in breathing after a long use. These types of problems can be eliminated with the custom-made masks since they have filters close to both the mouth and nostrils. In future, it is planned to further improve these masks and to neutralize the viruses in the inhaled air.

PCL has wide applicability in various fields, particularly as scaffolds in tissue engineering due to its good biological and physical properties. It also fulfils critical design requirements such as biocompatibility in order to successfully integrate living cells in the scaffold structure [29, 30].

In addition, the custom-made mask design fits perfectly on the face. However, with these masks, the risk of moisture formation is higher due to close contact. This humidity situation will be addressed in future studies, and a solution will be tried to be found.

\section{Conclusion}

In this study, a custom-made mask design was developed to prevent infection of the virus. The computer-aided simulations were performed using both classical and custommade mask and their performance was compared to prevent from Covid-19 and other infections diseases. The custommade mask design was found to be more effective than classical masks. The developed FEA model is beneficial in limiting the number of experiments required to model these type of processes. In future, we are planning to determine the effect of different mask materials.

\section{References}

[1] 1. Lu C-w, Liu X-f, and Jia Z-f, The Lancet. 395 (2020) e39.

[2] 2. Belser JA, Rota PA, Tumpey TM, Microbiology and Molecular Biology Reviews. 77 (2013) 144.

[3] To KK-W, Tsang OT-Y, Yip CC-Y, Chan K-H, Wu T-C, Chan JM-C, Leung W-S, Chik TS-H, Choi CY-C, Kandamby DH, Lung DC, Tam AR, Poon RW-S, Fung AY-F, Hung IF-N, Cheng VC-C, Chan JF-W, and Yuen K-Y, Clinical Infectious Diseases. 71 (2020) 841.

[4] 4. Holshue ML DC, and Lindquist S, N Engl J Med 382 (2020 ) 929.

[5] 5. Xiao F, Tang M, Zheng X, Liu Y, Li X, and Shan H, Gastroenterology. 158 (2020) 1831.

[6] 6. Zhang W, Du R-H, Li B, Zheng X-S, Yang X-L, Hu B, Wang Y-Y, Xiao G-F, Yan B, Shi Z-L, and Zhou P, Emerging Microbes \& Infections. 9 (2020) 386.

[7] 7. Harrel SK, and Molinari J, The Journal of the American Dental Association. 135 (2004) 429.

[8] 8. Liu L, Wei Q, Alvarez X, Wang H, Du Y, Zhu H, Jiang H, Zhou J, Lam P, Zhang L, Lackner A, Qin C, and Chen Z, Journal of Virology. 85 (2011) 4025. 
[9] 9. Cleveland JL, Gray SK, Harte JA, Robison VA, Moorman $\mathrm{AC}$, and Gooch BF, The Journal of the American Dental Association. 147 (2016) 729.

[10] Gok K, Measurement. 75 (2015) 57.

[11] Gok K, Inal S, Gok A, and Pinar AM, Journal of the Brazilian Society of Mechanical Sciences and Engineering. (2016) 1.

[12] 12. Inal S, Taspinar F, Gulbandilar E, and Gok K, The International Journal of Medical Robotics and Computer Assisted Surgery. 11 (2015) 95.

[13] 13. Erdem M, Gok K, Gokce B, and Gok A, Journal of Mechanics in Medicine and Biology. 17 (2016) 1750016.

[14] Gok K, Gok A, and Kisioglu Y, The International Journal of Advanced Manufacturing Technology. (2014) 1.

[15] Afsar E, Taspinar F, Calik BB, Ozkan Y, and Gok K, Journal of the Brazilian Society of Mechanical Sciences and Engineering. (2016) 1.

[16] 16. Gok K, Taspinar F, Inal S, and Gulbandilar E, Biomedical Engineering: Applications, Basis and Communications. 27 (2015) 1550030.

[17] 17. Sun P, Zeng D, Li X, Yang L, Li L, Chen Z, and Chen F, Symmetry. 12 (2020) 376.

[18] Li L, Xia Z, Jiang X, Ma Y, Roli F, and Feng X. 3D Face Mask Presentation Attack Detection Based on Intrinsic Image Analysis2019.

[19] 19. Jia S, Guo G, and Xu Z, Pattern Recognition. 98 (2020) 107032.

[20] Swennen GRJ, Pottel L, and Haers PE, International Journal of Oral and Maxillofacial Surgery.
[21] 21. Tarfaoui M, Nachtane M, Goda I, Qureshi Y, and Benyahia $\mathrm{H}$, The International Journal of Advanced Manufacturing Technology. 110 (2020) 2913.

[22] 22. Agrawal A, and Bhardwaj R, Physics of Fluids. 32 (2020) 101704.

[23] Gandhi P, Bafna R, Arabale G, Engineer S, and Phadke S, Transactions of the Indian National Academy of Engineering. (2020) 1.

[24] Sarkar S, Mukhopadhyay A, Sen S, Mondal S, Banerjee A, Mandal P, Ghosh R, Megaridis CM, and Ganguly R, Transactions of the Indian National Academy of Engineering. (2020) 1.

[25] Custom 3D printed Mask. Wasp.

[26] AnsysWorkbench. AnsysWorkbench Material Library. 2020.

[27] 27. Narayanan LK. Empirical and Finite Element Modeling of Structural and Mechanical Properties of 3D Bioplotted PCL Scaffolds. Raleigh, North Carolina: North Carolina State University; 2017.

[28] 28. Duck FA. Chapter 5 - Mechanical Properties of Tissue. In: Duck FA, editor. Physical Properties of Tissues. London: Academic Press; 1990. p. 137.

[29] 29. Labet M, Thielemans W, Chemical Society Reviews. 38 (2009) 3484.

[30] 3D Printing and Additive Manufacturing. 3 (2016) 106.

Publisher's Note Springer Nature remains neutral with regard to jurisdictional claims in published maps and institutional affiliations. 